\title{
Finite Element Method for Static Problems of Cubic Quasicrystals
}

\author{
L.-Z. YANG ${ }^{a, b}$, F.-M. $\mathrm{HE}^{c}$ AND Y. GAO ${ }^{a, *}$
}

${ }^{a}$ College of Science, China Agricultural University, Beijing 100083, China

${ }^{b}$ College of Engineering, China Agricultural University, Beijing 100083, China

${ }^{c}$ Sichuan Hydropower Investment and Management Group Ltd, 611130, China

We put forward a finite element method for static problems of cubic quasicrystals by variation of a suitable general potential functional. As an example we study a quasicrystal column containing a penny-shaped crack. The comparison with analytical results shows that the precision and efficiency of the numerical solution are satisfactory. The procedure can be used to solve more complicated boundary value problems and can be extended towards more sophisticated methods of crack tip loading analysis.

DOI: 10.12693/APhysPolA.126.471

PACS: $61.44 . \mathrm{Br}$

\section{Introduction}

A cubic quasicrystal is an important instance of a three-dimensional (3D) quasicrystal (QC). It is a $3 \mathrm{D}$ solid structure quasiperiodic in three orthogonal directions that supports simultaneously phonon and phason fields [1]. Analytical solutions for plane or 3D problems of these QCs with dislocations or cracks under simple boundary conditions have been obtained in terms of several methods such as Green's functions and displacement potentials [2, 3]. However, due to the large number of field variables and field equations involved in the elasticity theory of 3D QCs analytical solutions for 3D complex boundary value problems meet with tremendous difficulties.

In this paper, a finite element algorithm for static problems of cubic quasicrystals is derived from the variation of a kind of general potential function for QCs. A quasicrystal column containing a penny-shaped crack is investigated to verify the validity of the numerical method.

\section{Basic equations}

Within the theory of elasticity of QCs, displacements are denoted as $u_{i}(i=1,2,3)$ in the phonon field and $w_{i}$ in the phason field, both of which depend on the coordinate $x$ in the real space. The phonon strain $\varepsilon_{i j}$ and phason strain $w_{i j}$ of cubic QCs are given by the following equations:

$$
\varepsilon_{i j}=\left(\partial_{j} u_{i}+\partial_{i} u_{j}\right) / 2, \quad w_{i j}=\left(\partial_{j} w_{i}+\partial_{i} w_{j}\right) / 2,
$$
where $\partial_{j}=\partial / \partial x_{j}$, and the subscripts $j=1,2,3$.

The phonon stress $\sigma_{i j}$ and phason stress $H_{i j}$ are respectively given by linear elasticity of cubic QCs [4]. We arrange the stress components in the following vector as:

$$
\begin{gathered}
\left(\begin{array}{c}
\sigma_{i j} \\
H_{i j}
\end{array}\right)=\left\{\sigma_{11}, \sigma_{22}, \sigma_{33}, \sigma_{23}, \sigma_{31}, \sigma_{12},\right. \\
\left.H_{11}, H_{22}, H_{33}, H_{23}, H_{31}, H_{12}\right\}^{\mathrm{T}},
\end{gathered}
$$

\footnotetext{
*corresponding author; e-mail: gaoyangg@gmail.com
}

and the corresponding strain component can also be arranged according to the same order as

$$
\begin{gathered}
\left(\begin{array}{c}
\varepsilon_{i j} \\
w_{i j}
\end{array}\right)=\left\{\varepsilon_{11}, \varepsilon_{22}, \varepsilon_{33}, \gamma_{23}, \gamma_{31}, \gamma_{12},\right. \\
\left.w_{11}, w_{22}, w_{33}, \eta_{23}, \eta_{31}, \eta_{12}\right\}^{\mathrm{T}},
\end{gathered}
$$

where the superscript $T$ represents the transpose, $\gamma_{i j}=$ $2 \varepsilon_{i j}(i \neq j)$, and $\eta_{i j}=2 w_{i j}(i \neq j)$. The constitutive relations of cubic QCs can be written as

$$
\left(\begin{array}{c}
\sigma_{i j} \\
H_{i j}
\end{array}\right)=[D]\left(\begin{array}{c}
\varepsilon_{i j} \\
w_{i j}
\end{array}\right),
$$

where $[D]=\left[\begin{array}{ll}{[C]} & {[R]} \\ {[R]^{\mathrm{T}}} & {[K]}\end{array}\right]$, and

$$
[C]=\left[\begin{array}{cccccc}
C_{11} & C_{12} & C_{12} & 0 & 0 & 0 \\
C_{12} & C_{11} & C_{12} & 0 & 0 & 0 \\
C_{12} & C_{12} & C_{11} & 0 & 0 & 0 \\
0 & 0 & 0 & C_{44} & 0 & 0 \\
0 & 0 & 0 & 0 & C_{44} & 0 \\
0 & 0 & 0 & 0 & 0 & C_{44}
\end{array}\right],
$$$$
[R]=\left[\begin{array}{cccccc}
R_{1} & R_{2} & R_{2} & 0 & 0 & 0 \\
R_{2} & R_{1} & R_{2} & 0 & 0 & 0 \\
R_{2} & R_{2} & R_{1} & 0 & 0 & 0 \\
0 & 0 & 0 & R_{3} & 0 & 0 \\
0 & 0 & 0 & 0 & R_{3} & 0 \\
0 & 0 & 0 & 0 & 0 & R_{3}
\end{array}\right]
$$

$$
[K]=\left[\begin{array}{cccccc}
K_{11} & K_{12} & K_{12} & 0 & 0 & 0 \\
K_{12} & K_{11} & K_{12} & 0 & 0 & 0 \\
K_{12} & K_{12} & K_{11} & 0 & 0 & 0 \\
0 & 0 & 0 & K_{44} & 0 & 0 \\
0 & 0 & 0 & 0 & K_{44} & 0 \\
0 & 0 & 0 & 0 & 0 & K_{44}
\end{array}\right]
$$

$C_{11}, C_{12}, C_{44}$ represent the elastic constants in phonon field, $K_{11}, K_{12}, K_{44}$ are the elastic constants in phason 
field, and $R_{1}, R_{2}, R_{3}$ are the phonon-phason coupling elastic constants.

The static equilibrium equations are

$$
\partial_{j} \sigma_{i j}+f_{i}=0, \quad \partial_{j} H_{i j}+g_{i}=0,
$$

where $f_{i}$ and $g_{i}$ are, respectively, the body forces in the phonon and phason fields.

For a boundary value problems in a specific domain $\Omega, \Gamma_{t}$ and $\Gamma_{u}$, respectively, are used to denote the stress and displacement boundaries. Then, the above formulae are defined in any interior point of the domain $\Omega$. At the boundaries $\Gamma_{t}$ and $\Gamma_{u}$, the stresses and displacements satisfy the following boundary conditions:

$$
\begin{aligned}
& \left\{\begin{array}{l}
\sigma_{i j} n_{j}=T_{i}, \\
H_{i j} n_{j}=h_{i},
\end{array}\left(x_{1}, x_{2}, x_{3}\right) \in \Gamma_{t},\right. \\
& \left\{\begin{array}{l}
u_{i}=\bar{u}_{i}, \\
w_{i}=\bar{w}_{i},
\end{array}\left(x_{1}, x_{2}, x_{3}\right) \in \Gamma_{u},\right.
\end{aligned}
$$

where $T_{i}$ and $h_{i}$, respectively, are the traction vectors in the phonon and phason fields at the boundary $\Gamma_{t}, n_{j}$ is the unit outward normal vector at any point of the boundary $\Gamma_{t}$, and $\bar{u}_{i}$ and $\bar{w}_{i}$ are the given displacements at the boundary $\Gamma_{u}$.

\section{Finite element formulation for static elasticity of cubic QCs}

In Ref. [1], Fan has extended the minimum potential energy principle of classical elasticity to describe the elasticity of QCs. For sufficiently smooth boundaries and if all $u_{i}$ and $w_{i}$ satisfy Eq. (2.1) and displacement boundary conditions Eq. (2.6), the energy functional of cubic QCs reads

$$
\begin{aligned}
\Pi & =\int_{\Omega}\left(\begin{array}{c}
\varepsilon_{i j} \\
w_{i j}
\end{array}\right)[D]\left\{\varepsilon_{i j}, w_{i j}\right\} \mathrm{d} \Omega \\
& -\int_{\Omega}\left(f_{i} u_{i}+g_{i} w_{i}\right) \mathrm{d} \Omega-\int_{\Gamma_{t}}\left(T_{i} u_{i}+h_{i} w_{i}\right) \mathrm{d} \Gamma .
\end{aligned}
$$

Divide the $\mathrm{QC}$ body in the domain $\Omega$ into $M$ sub-domains or elements $\Omega^{(m)}$, where the superscript $m$ is the element number and $m=1, \ldots, M$, there is $\Omega=$ $\sum_{m=1}^{M} \Omega^{(m)}$. The degree of freedom of every node is six, that is $\{u\}=\left\{u_{1}, u_{2}, u_{3}, w_{1}, w_{2}, w_{3}\right\}^{\mathrm{T}}$.

Applying the variation of the discrete form of the functional $\Pi^{*}$, we obtain the following algebraic system of equations:

$$
[A]\{\tilde{u}\}=[F],
$$

where

$$
\begin{aligned}
{[A] } & =\sum_{m=1}^{M} \int_{\Omega^{(m)}}[B]^{\mathrm{T}}[D][B] \mathrm{d} \Omega, \quad\left[B_{\alpha}\right]=[L]\left[I_{\alpha}\right], \\
{[F] } & =\sum_{m=1}^{M}\left(\int_{\Omega^{(m)}}[I]^{\mathrm{T}}\left\{\begin{array}{l}
f^{(m)} \\
g^{(m)}
\end{array}\right\} \mathrm{d} \Omega\right. \\
& \left.+\int_{\Gamma_{t}^{(m)}}[I]^{\mathrm{T}}\left\{\begin{array}{l}
T^{(m)} \\
h^{(m)}
\end{array}\right\} \mathrm{d} \Gamma\right)
\end{aligned}
$$

and $\{\tilde{u}\}=\left\{\left\{\tilde{u}^{(1)}\right\},\left\{\tilde{u}^{(2)}\right\}, \ldots\left\{\tilde{u}^{(N)}\right\}\right\}^{\mathrm{T}}$ is the displacement vector of all nodes in the domain $\Omega, N$ is the total number of nodes, $\left[I_{\alpha}\right]$ is the element interpolation matrix, $\alpha$ is the node number, and $[L]$ is the element strain differential operator matrix, i.e.,

$$
\begin{aligned}
{\left[I_{\alpha}\right]=\left[\begin{array}{cccccc}
I_{\alpha} & 0 & 0 & 0 & 0 & 0 \\
0 & I_{\alpha} & 0 & 0 & 0 & 0 \\
0 & 0 & I_{\alpha} & 0 & 0 & 0 \\
0 & 0 & 0 & I_{\alpha} & 0 & 0 \\
0 & 0 & 0 & 0 & I_{\alpha} & 0 \\
0 & 0 & 0 & 0 & 0 & I_{\alpha}
\end{array}\right], } \\
{[L]=\left[\begin{array}{cccccccccccc}
\partial_{1} & 0 & 0 & 0 & \partial_{3} & \partial_{2} & 0 & 0 & 0 & 0 & 0 & 0 \\
0 & \partial_{2} & 0 & \partial_{3} & 0 & \partial_{1} & 0 & 0 & 0 & 0 & 0 & 0 \\
0 & 0 & \partial_{3} & \partial_{2} & \partial_{1} & 0 & 0 & 0 & 0 & 0 & 0 & 0 \\
0 & 0 & 0 & 0 & 0 & 0 & \partial_{1} & 0 & 0 & 0 & \partial_{3} & \partial_{2} \\
0 & 0 & 0 & 0 & 0 & 0 & 0 & \partial_{2} & 0 & \partial_{3} & 0 & \partial_{1} \\
0 & 0 & 0 & 0 & 0 & 0 & 0 & 0 & \partial_{3} & \partial_{2} & \partial_{1} & 0
\end{array}\right]^{\mathrm{T}} }
\end{aligned}
$$

The stress fields of cubic QCs in the $m$-th element can be evaluated at the Gauss integral points through the element node displacement components as follows

$$
\left(\begin{array}{l}
\sigma_{i j} \\
H_{i j}
\end{array}\right)^{(m)}=[D][B]\left\{\tilde{u}^{(m)}\right\} .
$$

In our computations, we use 20-node brick elements and 27 Gaussian points to perform the integrations in Eq. (3.3).

\section{A column containing a penny-shaped crack subjected to a tension}

Consider a QC column containing a penny-shaped crack subjected to a tension $P_{0}$ as shown in Fig. 1a, where the radius of crack $a$ is $1 \mathrm{~mm}$, the radius of the column is $20 \mathrm{~mm}$, the height is $60 \mathrm{~mm}$, and $P_{0}=0.1 \mathrm{GPa}$. Due to the symmetry of the specimen, only the upper $1 / 4$ part has to be modeled, and the finite element model is shown in Fig. 1b. A layer of elements with $0.1 \mathrm{~mm}$ height is placed at the bottom of the model in order to conveniently analyze the stress intensity factor of the crack. The elements related to the crack tip are degenerated in terms of singular quarter-point brick elements [5]. The boundary condition of the upper surface is $\sigma_{z}=P_{0}$. Other surfaces of the model are processed according to symmetry of the column.

Because elastic constants for cubic QCs are not available presently [6], the material parameters are assumed as follows: $C_{11}=4 \mathrm{GPa}, C_{12}=2 \mathrm{GPa}, C_{44}=3 \mathrm{GPa}$, $R_{1}=-1 \mathrm{GPa}, R_{2}=0.7 \mathrm{GPa}, R_{3}=0.5 \mathrm{GPa}, K_{1}=$ $10 \mathrm{GPa}, K_{2}=-7 \mathrm{GPa} K_{3}=60 \mathrm{GPa}$.

Due to the symmetry, the stress and displacement fields in every vertical cross-section containing the axis $z$ are the same. The phonon stress ratio $\sigma_{z} / P_{0}$ at the Gauss integration points of the element layer in the plane $x=0$ is given in Fig. 2a. The stress ratio at the crack tip is 26.6. Along with the increase of the ratio $(r-a) / a$, 

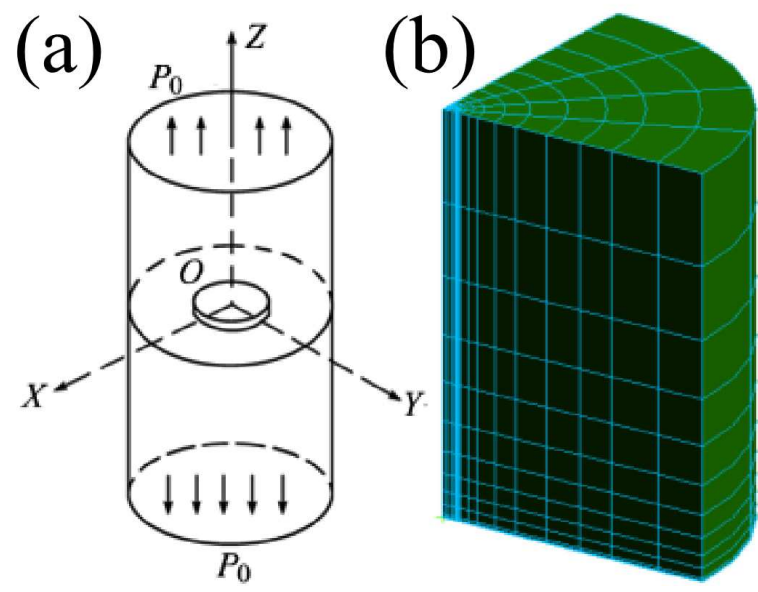

Fig. 1. Penny-shaped crack subjected to a tension in a cubic QC.

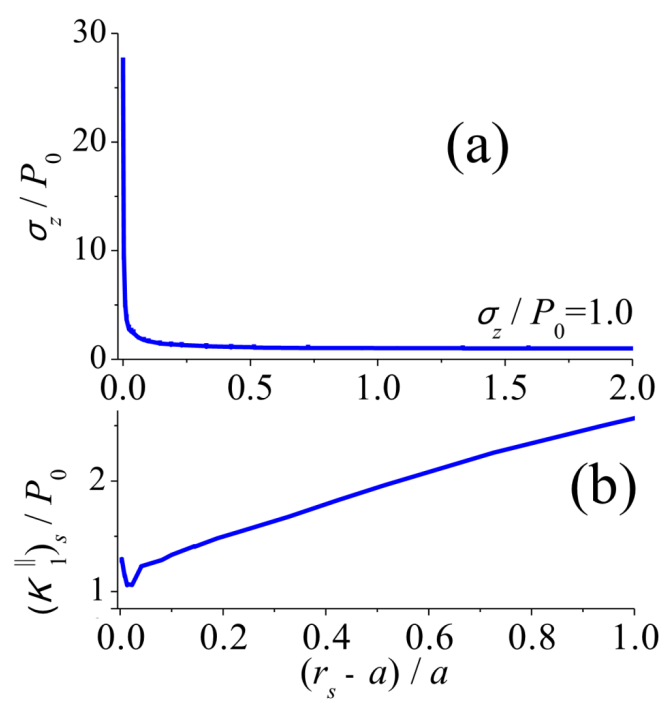

Fig. 2. (a) Phonon stress ratio $\sigma_{z} / P_{0}$; (b) stress intensity factor $\left(K_{1}^{\|}\right)_{s} / P_{0}$ from different Gauss points (unit: $\sqrt{\mathrm{mm}})$.

the stress ratio decreases rapidly and finally approaches 1.0 which satisfies the stress boundary condition. From Ref. [3], the phonon stress intensity factor for a penny-crack under a tension yields

$$
K_{\mathrm{I}}^{\|}=\lim _{r \rightarrow a^{+}}\left[\sqrt{2 \pi(r-a)} \sigma_{z}(r, 0)\right]=2 \sqrt{\pi a} P_{0} / \pi,
$$

where the last item is only valid for an infinite domain. Because the boundary of the column is very far from the crack, Eq. (4.1) is suitable for the column. Substituting $a$ and $P_{0}$ into Eq. (4.1), the stress intensity factor is $0.1128 \mathrm{MPa} \sqrt{\mathrm{mm}}$. On the other hand, the least-squares method [7] based on the stresses $\sigma_{z}$ at element Gauss points is implemented to calculate the stress intensity factor from numerical solutions by the following equation:

$$
\begin{aligned}
& K_{1}^{\|} \approx \frac{\sum\left(r_{s}-a\right) \sum\left(r_{s}-a\right)\left(K_{1}^{\|}\right)_{s}-\sum\left(r_{s}-a\right) \sum\left(K_{1}^{\|}\right)_{s}}{\left[\sum\left(r_{s}-a\right)\right]^{2}-Q \sum\left(r_{s}-a\right)^{2}}, \\
& \left(K_{1}^{\|}\right)_{s}=\sqrt{2 \pi\left(r_{s}-a\right)}\left(\sigma_{z}\right)_{s},
\end{aligned}
$$

where the subscript $s$ denotes the number of the Gauss point, and $s=1,2, \ldots, Q$ (Fig. $2 \mathrm{~b}$ ) gives the relations of $\left(K_{1}^{\|}\right)_{s} / P_{0}$ and $\left(r_{s}-a\right) / a$. The Gauss points in the domain $0.05<\left(r_{s}-a\right) / a<0.4$ are chosen to determine the stress intensity factor $K_{1}^{\|}$. The intensity factor is obtained as $0.1149 \mathrm{MPa} \sqrt{\mathrm{mm}}$. Compared to the analytical value $0.1128 \mathrm{MPa} \sqrt{\mathrm{mm}}$, the corresponding error is $1.9 \%$, confirming the accuracy of the numerical calculations.

\section{Conclusions}

Based on the variation of the general potential function of QCs, the 3D finite element formulation for cubic QCs is developed in this paper. The reliability of the procedure is verified by a column containing a penny-crack subjected to a tension. From the comparison of the analytical and numerical stress intensity factors, it can be seen that the numerical solutions are consistent with the analytical results. The procedure can be used to solve more complicated boundary value problems and can be extended towards more sophisticated methods of crack tip loading analysis.

\section{Acknowledgments}

The work is supported by the National Natural Science Foundation of China (No. 11172319) and Chinese Universities Scientific Fund (No. 2011JS046 and No. 2013BH008).

\section{References}

[1] T.Y. Fan, Mathematical Theory of Elasticity of Quasicrystals and Its Applications, Springer-Verlag, Berlin 2011.

[2] Y. Gao, A. Ricoeur, L L. Zhang, Phys. Lett. A 375, 2775 (2011).

[3] W.M. Zhou, T.Y. Fan, Chin. Phys. 9, 294 (2000).

[4] W.G. Yang, R.H. Wang, D.H. Ding, C.Z. Hu, Phys. Rev. B 48, 6999 (1993).

[5] R.D. Henshell, K G. Shaw, Int. J. Numer. Meth. Eng. 9, 495 (1975).

[6] S.H. Ju, Int. J. Numer. Meth. Eng. 43, 1437 (1998).

[7] T.Y. Fan, Engineering 5, 407 (2013). 